\title{
The concavity effect is a compound of local and global effects
}

\author{
Joachim Vandekerckhove, Sven Panis, and Johan Wagemans \\ University of Leuven, Leuven, Belgium
}

\begin{abstract}
Using a change detection paradigm, Barenholtz, Cohen, Feldman, and Singh (2003) found that changes in concave regions of a contour are more easily detected than changes in convex regions. In a series of three experiments, we investigated this concavity effect using the same paradigm. We observed the effect in wirelike stimuli as well as in silhouettes (Experiment 1) and in complex, smoothed images as opposed to angular polygons (Experiment 2). We also observed a systematic effect of the magnitude of the change (Experiment 1). Furthermore, we find that the effect cannot be attributed to either local or global processing effects, but rather to a combination of both "mere" concaveness and an effect due to changes in the perceived part structure of the stimulus object (Experiment 3). For our data analysis, we used a nonparametric bootstrap method, which greatly increases sensitivity (compared to more traditional analyses like ANOVA).
\end{abstract}

Mathematically, objects are commonly described by the shape and location of their boundaries - the lines or surfaces where the inside of the object ends and the outside world begins. In general, objects have little to define them but their edges. Accordingly, the visual system seems to process edges first, and derive surface information second (Lee, 2003; see also Marr, 1982). Interesting questions then arise: What other information is there in edges? What can this tell us about representation of visual shape?

In recent publications, much attention has been given to the issue of information content in object edges, both using an empirical (Barenholtz et al., 2003; De Winter \& Wagemans, 2004, in press-a, in press-b) and a theoretical approach (Feldman \& Singh, 2005; see also Resnikoff, 1985). The idea that some points on an edge are more important than others, on the other hand, is an old concept: Both Alhazen (1030/1989) and Attneave (1954) had noted that points of high curvature carry relatively more weight.

Interestingly, Feldman and Singh's (2005) theoretical account not only predicts a higher saliency for highcurvature regions, but also assigns a role to the sign of curvature. ${ }^{1}$ Because figure edges are typically closed curves, their total curvature (or turning angle) must be larger than zero (i.e., figure edges are, on average, convex). Therefore, for any region on a given contour, the prior expectation would be that it is (slightly) convex. A concave region is thus more "surprising" than a convex one; and that, in turn, means that its information content should be larger. Indeed, this prediction was empirically supported by Barenholtz et al. (2003). In their experiment, these authors used a procedure where observers were asked to compare two starlike polygons that were shown in rapid succession (250 msec each, with a $200-$ msec mask in between). In half of these trials, identical polygons were shown (nochange trials), and in the other half, a single vertex had been added or removed (change trials). Their critical independent variable was the type of change: Either the crucial vertex formed a concavity or it formed a convexity. As predicted by Feldman and Singh's theory, Barenholtz et al. (2003) found a "dramatic" advantage for concave changes compared to convex changes of equal magnitude.

Two explanations for this concavity effect have surfaced. On the one hand, there is the localist account, inspired by Feldman and Singh (2005; see above; see also Resnikoff, 1985), which entails that concavities carry more information in themselves, regardless of other stimulus properties. ${ }^{2}$ On the other hand, there is the globalist account, saying that concavities are more detectable because, in certain (identifiable) situations, they carry more weight in the formation of parts (De Winter \& Wagemans, 2006; Keane, Hayward, \& Burke, 2003; Koenderink, 1984). This distinction was also noted by Barenholtz et al. (2003), who wrote that a localist account would state that "concave sections of contour are inherently more salient, irrespective of the eventual role they play in shape decomposition" (Barenholtz et al., 2003, p. 7). A globalist account, on the other hand, would say that "concave regions are especially detectable because-and only insofar as - they influence the global decomposition of the shape into parts" (Barenholtz et al., 2003, p. 7). The latter hypothesis builds on such segmentation theories as the minima rule (Hoffman \& Richards, 1984) and the shortcut rule (Singh, Seyranian, \& Hoffman, 1999) or, more recently, the bracketing hypothesis (Bertamini \& Farrant, 2005). The minima rule states that human visual perception tends to decompose shapes into parts along lines of

J.Vandekerckhove, joachim.vandekerckhove@psy.kuleuven.be 
negative curvature minima. The short-cut rule states that this process divides silhouettes into parts using the shortest possible cuts, subject to two conditions: (1) at least one of the two potential boundary points has negative curvature, and (2) the cut crosses an axis of local symmetry. The bracketing hypothesis states that a convex vertex or a string of convexities in an edge signal a part, but only if they are bracketed by concave vertices. Bertamini and Farrant (2005) write, "We believe that what is salient does not depend on whether the vertex is convex or concave but whether it leads to a change in perceived part structure" (p. 44; our emphasis). Thus, the bracketing hypothesis is another theory to describe visual parsing where concavities may determine part structure because they "bracket" a cusp, but not "simply by virtue of being concavities" (Bertamini \& Farrant, 2005, p. 47). The validity of the localist and globalist accounts constitutes the major theme of the present study.

In Experiment 1, we set out to further validate and extend the paradigm used by Barenholtz et al. (2003). We will address two basic questions in this experiment. First, is the concavity effect a reliable (and stable) phenomenon? Second, does the concavity effect generalize to stimuli that consist of only a contour line ("wires")? This latter question is relevant because local processing of a thin piece of contour cannot give rise to any differential effects of concave versus convex changes, whereas global processing of the entire figure could explain the effect. The answer to this question could yield a first indication toward the larger question regarding the relative influence of local and global properties.

In Experiment 2, we investigate the robustness of the concavity effect and address only one question: does the concavity effect depend on simple, apparently irrelevant, properties of the stimulus shapes such as possessing straight edges and sharp angles?

In Experiment 3, we directly compare the two accounts of the concavity effect. By independently manipulating local and global features, we disentangle the localist and globalist accounts in a single design.

\section{Data Analysis Method}

In order to achieve maximally sensitive statistical tests, we used a bootstrap estimation method that, to our knowledge, has not been previously described in the literature in the context of signal detection.

The basic unit of data in any signal detection experiment is the bias-free $d^{\prime}$ measure. For purposes of statistical analysis, this measure carries both a large advantage and a large handicap. Its handicap is in the data reduction: within each condition in our design, a great many observations (an observer's responses on all trials within that condition) are reduced to a single data point $\left(d^{\prime}\right)$. Collapsing a large amount of data into what are essentially a few across-participant means inappropriately inflates measurement variance and thus greatly reduces the sensitivity of standard data-analytical techniques such as analysis of variance (for example, in our first experiment, we would be left with a mere thirteen data points in each condition, whereas we collected almost 10,000 data points!).
Fortunately, the $d^{\prime}$ statistic does provide more information than just a mean value. Using a simple bootstrap procedure (Monte Carlo analysis; Mooney \& Duval, 1993), it is possible to derive the distribution of $d^{\prime}$ within each condition, without making any distributional assumptions.

For a data set with $c$ conditions with $n$ data points each, we resample each condition within the original data set (i.e., from all trials within each condition, draw $n$ trials with replacement) $n$ times and recalculate $d^{\prime}$ for each new data set. This technique can be thought of as the nonparametric generation of "equivalent experiments."

Using the distribution of $d^{\prime}$ thus established, it becomes possible to sample $n$ data points from this distribution. These sampled data points can then be used as raw data in familiar statistical designs and thus yield an estimate of, for example, $F$ statistics or measures of association and effect size (though other statistics, regardless of their mathematical complexity, could be used equally easily). Because this procedure yields only estimates, it should be repeated many times (e.g., 1,000 times) and averaged in order to reduce errors of estimation. ${ }^{3}$

In our current study, we used this bootstrap method to estimate effect size statistics in analyses of variance and Student's $t$ tests. Due to the large amount of data used in each simulated sample, all effects reached significance at conventional levels (all $p \mathrm{~s}<10^{-10}$ ). ${ }^{4}$ For brevity, we report only $\omega^{2}$, a measure of association which expresses the proportion of variance in $d^{\prime}$ explained by each factor individually (Kirk, 1995). ${ }^{5}$ In other words, it has the same interpretation as the ubiquitous $R^{2}$ statistic. J. Cohen (1988) set as rules of thumb for $\omega^{2}$ that values of .01 are "small," .06 is "medium," and .15 is "large."

Throughout, we use $d^{\prime}$ as dependent measure. However, our change-detection paradigm is not ideally suited for this statistic. Because it is not possible to define different false-alarm rates for each condition in this paradigm, it becomes impossible to correct for response bias with standard psychophysical logic (i.e., within each condition separately). Hence, in contrast with other applications, $d^{\prime}$ is not a fully bias-free measure here. Nevertheless, we opted to use $d^{\prime}$ because of its desirable properties for data-analytical purposes (e.g., as opposed to percentage-correct statistics, it can be assumed to follow a normal distribution, and it is possible to analytically estimate confidence intervals).

\section{EXPERIMENT 1}

Barenholtz et al. (2003) suggest that the change detection task provides a powerful tool to study different aspects of visual representation. An obvious example would be to use the concavity effect to study perceptual Gestalt properties such as closure (because, logically, a stimulus must be perceived as a closed shape before the distinction concave/convex can be made). However, the silhouette stimuli used by Barenholtz et al. are, by their nature, always closed - because their edge is implicitly given by an abrupt change of color or contrast - and hence unsuitable for research concerning closure. Then again, we cannot merely assume that the concavity effect will remain present in "empty" shape stimuli ("wires"; Bertamini \& 
Farrant, 2006). Thus, we designed our first experiment to investigate the advantage for concavities in wire stimuli.

As mentioned in the introduction above, these wire stimuli are interesting for a second reason, because there are in principle two very different ways in which the visual system can process such a stimulus. On the one hand, it could be perceived as literally a wire - an elongated object that is curved in on itself and connects to its own "tail." On the other hand, it could be perceived not as an object in its own right, but as the 2-D contour of an object which occupies the entire encompassed area. This distinction is important, because it fits well with the localist/globalist debate: If the stimuli are perceived as wires, local processing cannot distinguish insides from outsides, consequently it cannot distinguish convexities from concavities. These distinctions can in fact be made only when the stimuli are perceived as outlines of global shapes. In this sense, obtaining the concavity effect with wire stimuli would provide a first indication of more global processing. In Experiment 1, these two accounts (perception of a wire or perception of an object contour) can be disentangled, since adding, for example, a concavity to a wire stimulus also implies adding a convexity on the other side. ${ }^{6}$ If that is so, then no difference in detectability between concave or convex changes should present itself. If, however, processing is more global, adding a concavity should be perceived as adding a concavity to a 2-D figure, and the concavity effect should persist.

Experiment 1 closely mirrored the experiment reported in Barenholtz et al. (2003), with the addition of a single variable: Stimuli were either wires or silhouettes. Also, the magnitude of each change was explicitly entered as an independent variable with four equally spaced levels.

In each trial, observers were shown, in succession, one randomly generated polygon, a mask, another polygon that was either identical or altered with respect to one vertex and a second mask. Their task was to indicate whether or not the two stimuli were identical in shape.

The main independent variables were change type (concavity or convexity) and stimulus type (wire or silhouette). Contrary to Barenholtz et al. (2003), we explicitly manipulated change magnitude (the absolute displacement of the changed vertex relative to the straight edge, measured in pixels over the radial axis to the center of the image), which we varied over four levels $(11.25,22.50,33.75$, or 45.00 pixels ). ${ }^{7}$ Together, this yielded a $2 \times 2 \times 4$ completely randomized design.

Two variables were considered control variables and balanced throughout the experiment, namely change direction (vertex added or removed) and block sequence (starting with a wires block or with a silhouettes block). A third variable, stimulus complexity (number of vertices was 9 to 12), was randomized between trials.

\section{Method}

Observers. Thirteen University of Leuven undergraduates served as naive observers for partial fulfillment of course requirements. All participants had normal or corrected-to-normal vision.

Stimuli and Hardware. Stimuli were generated with an algorithm that yielded simple nonsense polygons. For each stimulus, a set of $9-12$ polar coordinates was generated. We did this by constructing
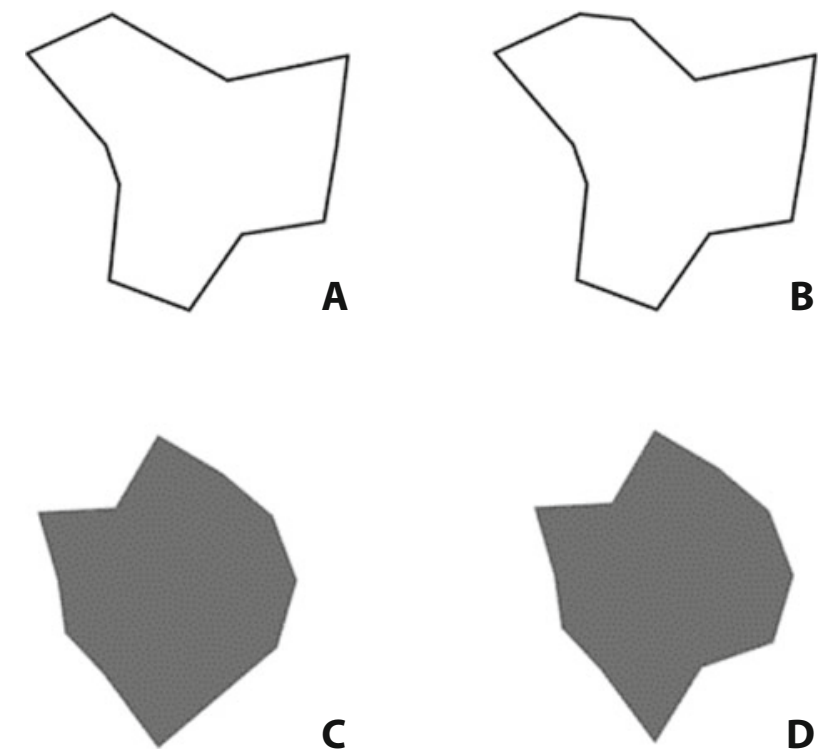

Figure 1. An example of two stimulus pairs for Experiment 1. (A) A wire base stimulus. (B) Stimulus A with an added convexity. (C) A silhouette base stimulus. (D) Stimulus $C$ with an added concavity.

a vector $\boldsymbol{\theta}$ of $n$ equally spaced angles (with steps of $2 \pi / n$ ) and generating a vector $\mathbf{r}$ of distances with lengths randomly chosen between $60 \%$ and $100 \%$ of the maximum allowed radius of the shape. Then, in each of the resulting polygons, one vertex was randomly chosen and removed. This was the "base" shape (Figure 1A). We then generated a second polygon by introducing a vertex (either a convexity or a concavity) of a fixed size $(11.25,22.50,33.75$, or 45.00 pixels displacement) where one had been removed in the base shape, by translating the vertex point over the radial axis (Figure 1B).

Figures were either rendered as wires or as silhouettes (all displayed in gray on a black background, at sufficient contrast to be clearly visible), and were smoothed through a 2-D Gaussian filter (with standard deviations of 1 pixel and covariance 0 ) in order to avoid coarse, serrated lines and edges. From an observer distance of $57 \mathrm{~cm}$, they measured between $3^{\circ}$ and $5^{\circ}$ of visual angle. A new set of stimuli was generated for each participant.

The mask stimuli that were presented after each figure consisted of a grid of 25 connected squares $(5 \times 5)$, each in a randomly selected shade of gray. The masks completely covered the region of the screen where the stimuli had appeared $\left(5.5^{\circ}\right.$ of visual angle).

The stimulus generation and experiment were performed using MATLAB (Version 5.1; The MathWorks) and the Psychophysics Toolbox extensions (Version 2.5; Brainard, 1997; Pelli, 1997), running on a Microsoft Windows XP Professional IBM-compatible PC with a Pentium IV $(2.8 \mathrm{GHz}) \mathrm{CPU}$ and a Dell E771p monitor with a resolution of $1,024 \times 768$ pixels and a $60-\mathrm{Hz}$ refresh rate.

Procedure. The experiment was run in a darkened room. The task was to detect whether a change had occurred or not. Observers were shown (1) a fixation cross for $167 \mathrm{msec}$; (2) one polygon for $250 \mathrm{msec}$; (3) a mask for $200 \mathrm{msec}$; (4) the second polygon for $250 \mathrm{msec}$; and (5) a mask until response. The experiment started with a short demonstration of the trial sequence and 48 practice trials, followed by eight blocks of 96 trials each, for a total of 768 experimental trials. Blocks alternatingly contained wire or silhouette stimuli, and each block contained six trials of each type (change/ no-change $\times$ change type $\times$ change magnitude), presented in randomized order. Observers were asked to indicate whether the two successively presented stimuli were identical or not by pressing one of two designated keys. They received auditory feedback after each trial, were allowed a pause after each block and had to pause after 

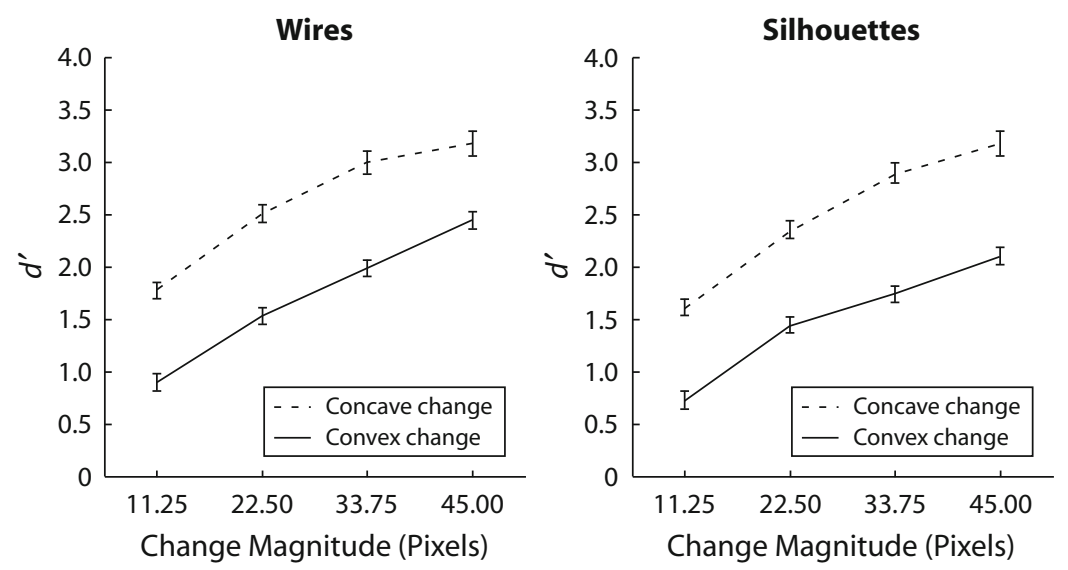

Figure 2. Results of Experiment 1. Error bars indicate $99 \%$ confidence intervals. (Left) Mean $d^{\prime}$ scores for the wire condition. (Right) Mean $d^{\prime}$ scores for the silhouette condition. In either case, performance increases with magnitude of change, and performance is higher for concave changes (dotted line) than for convex changes (full line). There is no appreciable difference between wires and silhouettes, and there are no strong interactions.

the fourth block. Participants were encouraged to reply accurately, rather than speedily.

\section{Results}

Using the bootstrap estimation method described above, we performed a 2 (change type) $\times 2$ (stimulus type) $\times 4$ (change magnitude) ANOVA. This yielded a very strong main effect of change type $\left(\omega^{2}=.404\right)$, but only a relatively small effect of stimulus type $\left(\omega^{2}=.026\right)$ and, importantly, their interaction was negligible $\left(\omega^{2}=.001\right)$. There was also a strong main effect of change magnitude $\left(\omega^{2}=.546\right)$, which did not show strong interactions with either of the other main variables or with their interaction (all $\omega^{2} \mathrm{~s}<.003$ ). The total $R^{2}$ (proportion of variance explained) was .986. See also Figure 2.

\section{Discussion}

The results of Experiment 1 largely speak for themselves. The goal of the experiment was to replicate the concavity effect, as described by Barenholtz et al. (2003), and to investigate if the effect would also emerge in wire stimuli. In the present design, the factor change type captures the concavity effect. Given the large advantage of concave over convex changes, we conclude that the concavity effect is strong. We also conclude that the effect appears equally strongly in wire and silhouette stimuli, and we interpret this as a first indication of holistic processing of shape (a globalist perspective).

Another interesting conclusion may be drawn from the present data. An intuitive explanation for the advantage for concavities would be that changes in concavities affect an area of the stimulus that is, on average, closer to the center of the shape and thus closer to the fixation point. However, such an effect would logically have to diminish as the magnitude of the change decreases, and we would then expect to find a strong interaction between change size and change type. Although the usual warnings regard- ing the accepting of null hypotheses apply, we believe our data allow us to reject this hypothesis.

\section{EXPERIMENT 2}

The stimuli used in our first experiment share many important qualities. One important shared quality is their angularity (shared by all our stimuli as well as those used by Barenholtz et al., 2003), which is of course a basic property of polygons. However, straight edges and sharp angles are not universal in natural objects. Moreover, tangent discontinuities (i.e., apexes) have previously been observed to contain powerful visual cues for shape perception (De Winter \& Wagemans, in press-a; Kristjansson $\&$ Tse, 2001) and recognition (Biederman, 1987). From a slightly different perspective, Hoffman and Singh (1997) state that "boundaries differ in salience: the higher curvature are more salient than the lower" (p. 54; "Hypothesis of normalized curvature"). Apexes can be said to have infinite curvature. In short, our stimulus sets have hitherto consisted of special shapes with special properties. In this experiment, we test the robustness of the concavity effect by removing the sharp angles and straight lines from our stimuli.

Experiment 2 was designed to be similar to Experiment 1. All observers were shown a randomly generated, smooth, closed curve (see Stimuli and Hardware, in the Method section, for details on these stimuli), followed by a mask, another such curve (either identical or altered with respect to one curvature extreme) and a second mask. The task was to indicate whether or not the two stimuli were identical.

The only important independent variable was change type (concavity or convexity). We did not use silhouette stimuli, nor was the magnitude of the change varied (it was kept constant at 22.50 pixels displacement).

One other variable, change direction (curvature extreme added or removed), was treated as a control variable and 
was balanced throughout the experiment. As in Experiment 1 , stimulus complexity (number of large extremes of curvature was 9 to 12), was randomized between trials.

\section{Method}

Observers. Eleven University of Leuven undergraduates, naive to the aim of the research, participated in this experiment and Experiment 3 for partial fulfillment of course requirements. ${ }^{8}$ All had normal or corrected-to-normal vision.

Stimuli and Hardware. Stimuli were generated with an algorithm that was based on the algorithm used to generate the simple nonsense polygons of Experiment 1. Again, for each stimulus, two sets of polar coordinates were generated, each describing a polygon: one with 9-12 vertices, the other with 8-11, but otherwise identical. The extra vertex in the changed shape consisted of a displacement of exactly 22.50 pixels (over the radial axis) from the straight edge variant.

A spline fitting procedure was applied in order to avoid straight lines and salient angles in the figure ${ }^{9}$ (see Figure 3 for an example). All figures were rendered as wires, displayed in gray on a black background and were smoothed through a customized anti-aliasing algorithm. A new set was generated for each observer. From an observer distance of $57 \mathrm{~cm}$, the images measured between $3^{\circ}$ and $5^{\circ}$ of visual angle.

The stimulus generation and experiment were performed using the same hardware and software as Experiment 1. We also made use of the Spline Toolbox (The MathWorks).

Procedure. The procedure for this experiment was similar to the one in Experiment 1, with the following exceptions. The experiment consisted of four blocks of 80 trials each, for a total of 320 experimental trials. There was also no practice session, as all observers had completed Experiment 3 shortly before participating in this experiment (see note 8). Each block contained twenty trials of each type (change/no-change $\times$ change type). Observers responded using one of two designated keys and received auditory feedback after each trial.

\section{Results}

In this experiment, we employed an exceedingly simple design, with only one variable of interest (change type). Using our bootstrap estimation method, we performed a Student's $t$ test to examine the difference between changes in concavities and changes in convexities (mean $d$ 's were 1.717 , and 1.416 , respectively). This difference was again very strong $\left(\omega^{2}=R^{2}=.541\right)$.

\section{Discussion}

In Experiment 2, we tested the robustness of the concavity effect, by using smoothed polygons rather than the angular, star-shaped ones used by Barenholtz et al. (2003) and in our own Experiment 1 . The concavity effect clearly and strongly persisted.

\section{EXPERIMENT 3}

In the introduction and in Experiment 1, two possible explanations of the concavity effect were put forward. There was the localist account, which says that concavities are more detectable because they are typically less frequent than convexities and their occurrence is therefore more "surprising" (Feldman \& Singh, 2005). If that is so, then the advantage for concavities is by virtue of being concave per se. Alternatively, some have suggested that changes in concavities are more detectable because of the more important role concavities play in the formation of parts (a globalist account; e.g., Bertamini \& Farrant, 2005; see also Bertamini \& Lawson, 2006; Hoffman \& Richards, 1984; Koenderink, 1984). That explanation would imply effects of the context in which a concavity or convexity occurs - its context could either be such that the part structure is affected by a change in one concavity, or it could be such that it is not. For example, a concavity added between two convexities splits one part into two, but a convexity in the same location (context) would not. Bertamini and Farrant (2005) found this context to be an important factor that can reduce the concavity effect to nonsignificant levels. However, in that study, Bertamini and Farrant had to accept a "null effect," and as we know, the absence of evidence is not evidence of absence. It remains possible that Bertamini and Farrant's aperture method is merely lacking in sensitivity (i.e., statistical power) because observers could see only part of the object - potentially leaving them with less stimulus information upon which to base a decision.

Here, we return to the established paradigm in order to disentangle the effect of concavities in themselves from their effect "in context." In Experiment 3, we generated polygons as in Experiment 1, but instead of merely adding or removing one vertex, in another condition an already existing vertex was increased or decreased, thereby retaining both part structure and context (because we neither add nor remove vertices, the sequence of vertices, and thus the "global" shape and all "contexts," is preserved in this condition). This manipulation closely resembles an old study from our lab presented at two conferences (Hanoulle, Waeytens, Wagemans, d'Ydewalle, \& Van Rensbergen, 1993; Wagemans, Hanoulle, Waeytens, \& d'Ydewalle, 1994 ) and included in a book chapter (Waeytens, Ha-
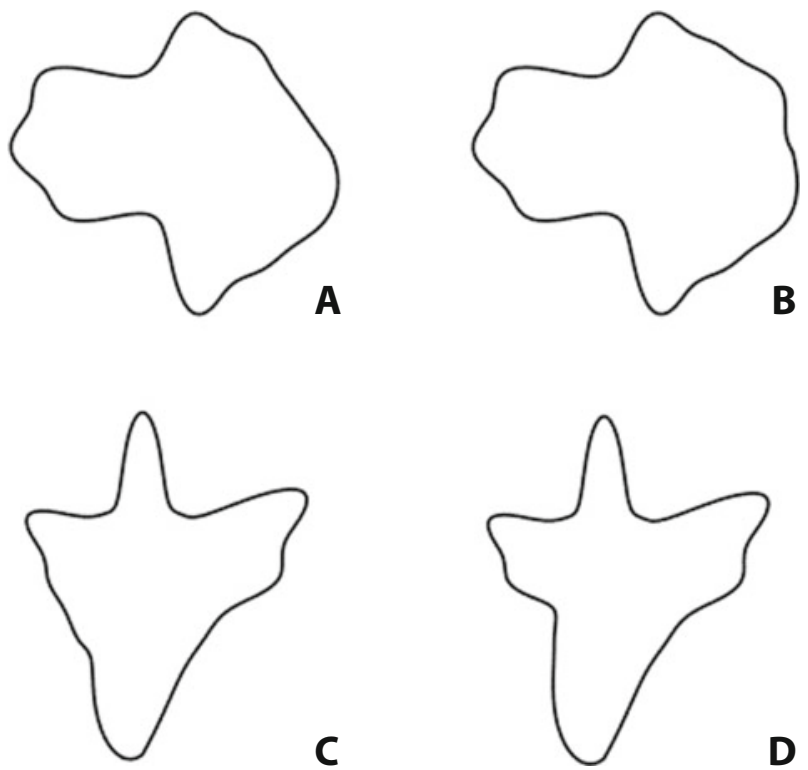

Figure 3. An example of two stimulus pairs for Experiment 2. (A) A base stimulus. (B) Stimulus A with an added convexity. (C) A second base stimulus. (D) Stimulus $C$ with an added concavity. 


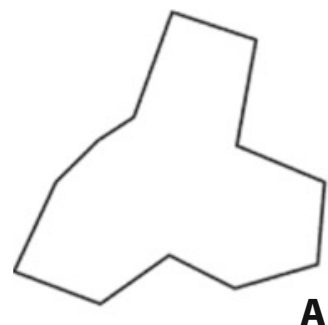

A
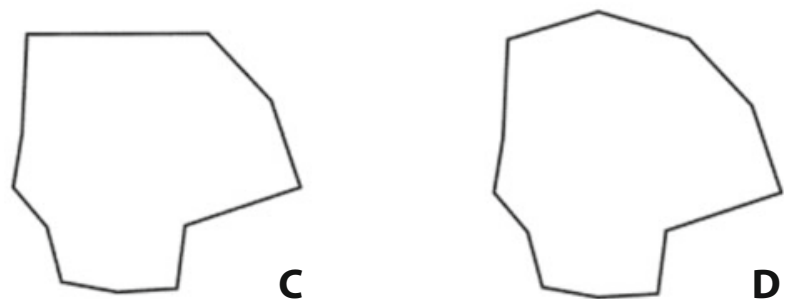

Figure 4. An example of two stimulus pairs for Experiment 3. (A) A base stimulus. (B) Stimulus A with a quantitative decrease in a concavity. (C) A second base stimulus. (D) Stimulus $C$ with a qualitative addition of a convexity.

noulle, Wagemans, \& d'Ydewalle, 1994), as well as the first experiment reported in E. Cohen, Barenholtz, Singh, and Feldman (2005). In the latter study, however, quantitative changes only were made (as opposed to both quantitative and qualitative). As a result, direct comparisons between local and global effects were not possible. E. Cohen et al.'s basic findings indicated that a quantitative change can indeed suffice for a concavity effect to occur.

\section{Method}

Observers. Experiment 3 was completed by the same observers as Experiment 2 (see note 8). Due to a technical malfunction, data for one participant were lost (final $n=10$ ).

Stimuli and Hardware. In this experiment, there was one new variable of interest: In the base stimulus, the crucial vertex was either completely absent or protruded by exactly 18.75 pixels (as compared with a straight edge). We call this variable the quality of the change (a qualitative difference, from nothing to a vertex, or a quantitative difference, from one vertex to a more/less extreme one; see Figure 4 for an illustration). Then, in the second stimulus the vertex was made more extreme by 18.75 pixels, resulting in a changed stimulus with a total displacement of either 18.75 or 37.5 pixels in the crucial vertex. The sequences base/changed (adding a vertex or increasing an existing vertex) and changed/base (removing a vertex or reducing an existing vertex) were counterbalanced across trials.

All figures were again rendered as wires, displayed in gray on a black background and were smoothed through the same anti-aliasing algorithm as in Experiment 2. From an observer distance of $57 \mathrm{~cm}$, the images again measured between $3^{\circ}$ and $5^{\circ}$ of visual angle. Each observer was tested with a new stimulus set.

The stimulus generation and experiment were performed using the same procedure, hardware and software as Experiment 1.

Procedure. The design of Experiment 3 was nearly identical to that of Experiment 1, with one variable of real interest, namely the change quality: Either a new vertex was introduced (or removed) or an existing one was increased (or decreased).
The procedure started with a short demonstration of the trial sequence and 40 practice trials, followed by eight blocks of 80 trials each, for a total of 640 experimental trials. Each block contained ten trials of each type (change/no-change $\times$ change type $\times$ change quality), presented in randomized order. Change magnitude was kept constant at 18.75 pixels displacement. Observers responded using one of two designated keys and received auditory feedback after each trial.

\section{Results}

We used our bootstrap method to perform a 2 (change type) $\times 2$ (change quality) ANOVA with $d^{\prime}$ as dependent variable. This revealed strong main effects of change type $\left(\omega^{2}=.499\right)$ and change quality $\left(\omega^{2}=.393\right)$, and there was a slight interaction between the two $\left(\omega^{2}=.060\right)$. Total $R^{2}$ was .959. See also Figure 5.

\section{Discussion}

From Experiment 3, several conclusions can be drawn. First, the large main effect of the quality of the change indicates an important role of the sequence of vertices in a polygon. This finding seems to corroborate Bertamini and Farrant's (2005) bracketing hypothesis. However, we also found a main effect of change type, which occurred both in the qualitative and in quantitative change conditions. Considering also that the interaction between these two variables was weak, we can conclude that an effect of concavities in itself also exists, contradicting Bertamini and Farrant's bracketing hypothesis.

Hence, while previous experiments may have found a concavity effect that was partly due to contamination by the bracketing effect, the advantage for concavities per se remains.

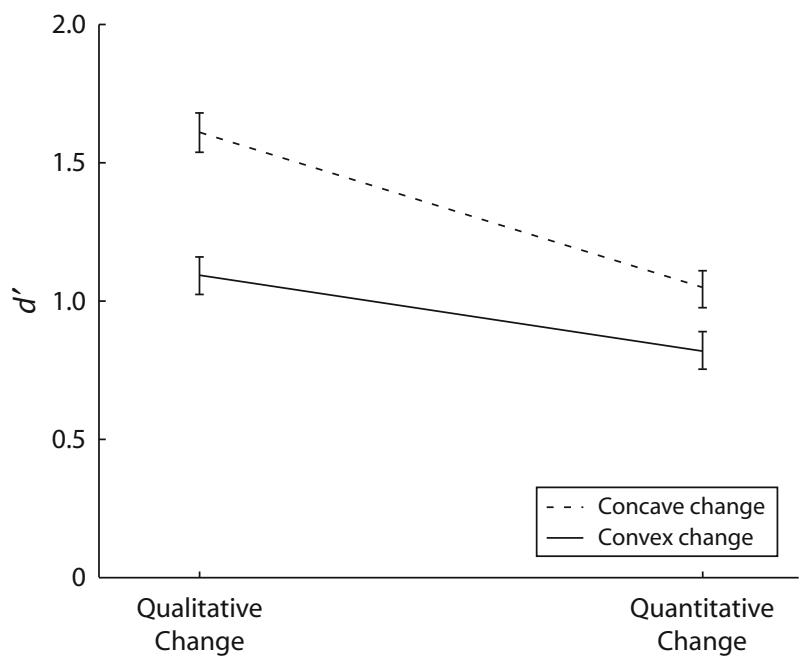

Figure 5. Results of Experiment 3. Error bars indicate 99\% confidence intervals. The dotted line indicates concave changes; the full line indicates convex changes. Both change type and change quality exert a strong main effect on $d^{\prime}$. There is a slight interaction. Importantly, an effect of concavities remains evident even when part structure is kept constant (in the quantitative change condition). 


\section{GENERAL DISCUSSION}

In Experiments 1-3, we built upon a procedure used by Barenholtz et al. (2003), who had experimentally established that changes in concavities are more easily detected than changes in convexities.

In Experiment 1, we used Barenholtz et al.'s change detection paradigm and (1) reestablished the base effect and (2) established that the advantage for concavities is also extant in wire stimuli (as opposed to the silhouettes used by Barenholtz et al.). A localist account cannot explain the concavity effect in these stimuli, since they do not possess local cues from which the distinction concave/convex can be made. In demonstrating the concavity effect in wire stimuli, we thus provide a first indication of global processing of shape.

In Experiment 2, we confirmed that the advantage for concavities also occurs in smooth stimuli (without sharp angles or straight edges), thereby confirming the robustness of the concavity effect in the face of a different type of stimulus.

In Experiment 3, we directly compared two theoretical explanations for the advantage for concavities. In one condition, we used stimuli whose part structure and context did not change. Even so, an effect of "mere" concaveness was found when part structure and context were kept constant. On the other hand, comparing change detection performance with those situations where part structure did change, it appeared that an independent advantage for changes in part structure also exists (as found by Bertamini \& Farrant, 2005). We conclude that, in contrast to the "bracketing" account of part segmentation both effects are real, and both contribute to the advantage for concavities.

However, we should note here that perhaps the dichotomy between local and global effects is too strong. In fact, we feel that strictly considering this as a dichotomy may cloud the issue, which is likely more complex than either/or. The results of our Experiment 3, we hope, may contribute in laying to rest this dichotomy, and direct attention toward the interplay between global and local effects. Rather than asking which of these two kinds of factors plays the critical role, we should now focus more on how they combine and interact. For example, the local advantage for concavities may be a visual heuristic that is useful because of the often important role of concavities on a global scale. Additionally, global and local effects may be inherently confounded: changes in the global structure of a figure may themselves influence the salience of local features (see also De Winter \& Wagemans, 2004, 2006, in press-a, in press-b).

As a general conclusion, we find that (1) the change detection paradigm has been reconfirmed as a valid method to investigate basic properties of shape perception such as the advantage for concavities, and (2) the advantage for concavities cannot be fully explained by either local or global influences, but is a compound effect.

\section{AUTHOR NOTE}

This research was supported by a research grant from the University Research Council (OT/00/007) and from the Fund for Scientific Re- search (FWO-Vlaanderen G.0189.02) to J.W. The research also forms part of a larger research program funded by the University Research Council (GOA-TBA/2005/03). The authors thank the participants of the 36th Meeting of the European Mathematical Psychology Group for helpful comments on the data analysis technique (Vandekerckhove, Panis, \& Wagemans, 2005), and three anonymous reviewers for helpful comments on previous versions of this article. Correspondence relating to this article may be sent to J. Vandekerckhove, University of Leuven, Department of Psychology, Tiensestraat 102, B-3000 Leuven, Belgium (e-mail: joachim.vandekerckhove@psy.kuleuven.be).

\section{REFERENCES}

Alhazen, I. (1989). Book of optics. In A. I. Sabra (Ed. \& Trans.), The Optics of Ibn al-Haytham (Vol. 1). London: Warburg Institute. (Original work published ca. 1030)

Attneave, F. (1954). Some informational aspects of visual perception. Psychological Review, 61, 183-193.

Barenholtz, E., Cohen, E., Feldman, J., \& Singh, M. (2003). Detection of change in shape: An advantage for concavities. Cognition, 89, $1-9$

Barenholtz, E., \& Feldman, J. (2003). Visual comparisons within and between object-parts: Evidence for a single-part superiority effect. Vision Research, 43, 1655-1666.

Bertamini, M., \& Farrant, T. (2005). Detection of change in shape and its relation to part structure. Acta Psychologica, 120, 35-54.

Bertamini, M., \& Farrant, T. (2006). The perceived structural shape of thin (wire-like) objects is different from that of silhouettes. Perception, 35, 1679-1692.

BERTAMINI, M., \& LAWSON, R. (2006). Visual search for a circular region perceived as a figure versus as a hole: Evidence of the importance of part structure. Perception \& Psychophysics, 58, 776-791.

BIEDERMAN, I. (1987). Recognition-by-components: A theory of human image understanding. Psychological Review, 94, 115-147.

Brainard, D. (1997). The Psychophysics Toolbox. Spatial Vision, 10, 433-436.

CHERNICK, M. (1999). Bootstrap methods: A practitioner's guide. New York: Wiley.

Cohen, E., Barenholtz, E., Singh, M., \& Feldman, J. (2005). What change detection tells us about the visual representation of shape. Journal of Vision, 5, 313-321.

CoHEN, J. (1988). Statistical power analysis for the behavioral sciences (2nd ed.). Hillsdale, NJ: Erlbaum.

Davison, A., \& Hinkley, D. (1997). Bootstrap methods and their application. Cambridge: Cambridge University Press.

De Winter, J., \& Wagemans, J. (2004). Contour-based object identification and segmentation: Stimuli, norms and data, and software tools. $B e-$ havior Research Methods, Instruments, \& Computers, 36, 604-624.

De Winter, J., \& Wagemans, J. (2006). Segmentation of object outlines into parts: A large-scale, integrative study. Cognition, 99, 275-325.

De Winter, J., \& Wagemans, J. (in press-a). The awakening of Attneave's sleeping cat: Identification of everyday objects on the basis of straight-line versions of outlines. Perception.

De Winter, J., \& Wagemans, J. (in press-b). Perceptual salience of points along the contour of everyday objects: A large-scale study. Perception \& Psychophysics.

EFron, B. (1979). Bootstrap methods: Another look at the jackknife. Annals of Statistics, 7, 1-26.

Efron, B., \& Tibshirani, R. (1993). An introduction to the bootstrap. Boca Raton, FL: CRC Press.

Feldman, J., \& Singh, M. (2005). Information along contours and object boundaries. Psychological Review, 112, 243-252.

Hanoulle, I., Waeytens, K., Wagemans, J., D'Ydewalle, G., \& Van Rensbergen, J. (1993, August). Representation and segmentation of shape based on curvature singularities. Poster presented at the 16th European Conference on Visual Perception (ECVP), Edinburgh.

Hoffman, D., \& Richards, W. (1984). Parts of recognition. Cognition, 18, 65-96.

Hoffman, D., \& Singh, M. (1997). Salience of visual parts. Cognition, 63, 29-78.

Keane, S., Hayward, W., \& Burke, D. (2003). Detection of three types of changes to novel objects. Visual Cognition, 10, 101-129. 
KIRK, R.E. (1995). Experimental design: Procedures for the behavioral sciences. Belmont, CA: Brooks/Cole.

KoenderinK, J. (1984). What does the occluding contour tell us about solid shape? Perception, 13, 321-330.

Kristjánsson, A., \& Tse, P. (2001). Curvature discontinuities are cues for rapid shape analysis. Perception \& Psychophysics, 63, 390-403.

LEE, T. (2003). Computations in the early visual cortex. Journal of Physiology, 97, 121-139.

MARR, D. (1982). Vision: A computational investigation into the human representation and processing of visual information. San Francisco: W. H. Freeman.

Mooney, C., \& Duval, R. (1993). Bootstrapping: A nonparametric approach to statistical inference. London: Sage.

Pelli, D. (1997). The VideoToolbox software for visual psychophysics: Transforming numbers into movies. Spatial Vision, 10, 437-442.

RESNIKOFF, H. (1985). The illusion of reality: Topics in information science. New York: Springer.

Singh, M., \& Hoffman, D. (2001). Part-based representations of visual shape and implications for visual cognition. In T. Shipley \& P. Kellman (Eds.), From fragments to objects: Grouping and segmentation in vision (Advances in Psychology, Vol. 130, pp. 401-459). New York: Elsevier.

Singh, M., Seyranian, G., \& Hoffman, D. (1999). Parsing silhouettes: The short-cut rule. Perception \& Psychophysics, 61, 636-660.

Vandekerckhove, J., Panis, S., \& Wagemans, J. (2005, September). An empirical measure of closure. Paper presented at the 36th Meeting of the European Mathematical Psychology Group, Padua.

Waeytens, K., Hanoulle, I., Wagemans, J., \& D'Ydewalle, G. (1994). Human representation of closed contours. In F. Dillen, I. Van de Woestijne, \& L. Verstraelen (Eds.), Geometry and topology of submanifolds VI (pp. 298-306). Singapore: World Scientific.

Wagemans, J., Hanoulle, I., Waeytens, K., \& D'Ydewalle, G. (1994). Are curvature singularities sufficient to represent shape? [Abstract]. Investigative Ophthalmology \& Visual Science, 35, 1918.

\section{NOTES}

1. There are different ways to determine the sign of curvature. In the context of 2-D figure perception, it is customary to assign negative curvature to regions that curve inward (i.e., concavities) and positive curvature to regions that curve outward (i.e., convexities).

2. We should note that we do not mean to imply that Feldman and Singh defend a strictly localist account. In fact, regarding perceived part structure, these authors have argued and shown that simply the local presence of negative minima is not sufficient (e.g., Barenholtz \& Feldman, 2003; Singh \& Hoffman, 2001).

3. Some helpful references on the topic of bootstrap methods are Efron and Tibshirani (1993; see also Efron, 1979), who explain the theoretical basis, and Mooney and Duval (1993), Chernick (1999), and Davison and Hinkley (1997), for more practical information.

4. Note that these small $p$ values are not due to a large number of simulated samples; in fact, we simulated exactly as many samples as there were data points in the original data set. The small $p$ values are the result of large differences between within-group and between-group variances of $d^{\prime}$ (i.e., large $F$ values). However, for the interpretation of our results, measures of association (e.g., proportion of variance explained) are more appropriate.

5. With bootstraps, it is sometimes interesting to report confidence intervals for bootstrapped statistics. However, the values for $\omega^{2}$ reported here show standard errors of estimation below reporting precision (below .001).

6. We are indebted to an anonymous reviewer for providing this insight.

7. We use pixels as standard measure throughout the paper. For reference, one pixel in our setup equaled $0.0349^{\circ}$ of visual angle. The use of fractional pixels may seem puzzling. During the stimulus generation, shapes were generated at much higher resolutions than a computer screen would allow. This high-resolution matrix was then fed into the anti-aliasing algorithm, which converted it into a smoothed grayscale image. This technique allowed us to present stimuli without serrated lines and edges, and provides a context in which it makes sense to use fractions of pixels.

8. It should be noted that these observers participated in Experiment 3 first, and in Experiment 2 shortly after that. This was done because we considered the possibility of carryover or learning effects to be more worrisome in that experiment than in this one.

9. In order to create a smoothly curved wire, a periodic cubic spline fit was applied to the $\mathbf{r}$ vector (which contained the distance of each vertex from the origin) of each polygon, yielding a smooth function with the previously defined number and sequence of extremes when converted into Cartesian coordinates. We boosted the sample rate of the $\mathbf{r}$ vector by interpolating three points between each pair of consecutive points, in a linear fashion. This preserved the correct number of curvature extremes and the overall resemblance with the original polygonal stimuli. MAT$\mathrm{LAB}$ code for constructing these stimuli (using the CSAPE function from the Spline Toolbox) is available upon request.

(Manuscript received January 20, 2006; revision accepted for publication April 16, 2007.) 